\title{
Pulse propagation in tapered granular chains: An analytic study
}

\author{
Upendra Harbola, ${ }^{1}$ Alexandre Rosas, ${ }^{2}$ Massimiliano Esposito, ${ }^{1,3}$ and Katja Lindenberg ${ }^{1}$ \\ ${ }^{1}$ Department of Chemistry and Biochemistry, and BioCircuits Institute, University of California-San Diego, La Jolla, \\ California 92093-0340, USA \\ ${ }^{2}$ Departamento de Física, CCEN, Universidade Federal da Paraíba, Caixa Postal 5008, João Pessoa 58059-900, PB, Brazil \\ ${ }^{3}$ Center for Nonlinear Phenomena and Complex Systems, Université Libre de Bruxelles, Code Postal 231, \\ Campus Plaine, B-1050 Brussels, Belgium
}

(Received 25 May 2009; published 15 September 2009)

\begin{abstract}
We study pulse propagation in one-dimensional tapered chains of spherical granules. Analytic results for the pulse velocity and other pulse features are obtained using a binary collision approximation. Comparisons with numerical results show that the binary collision approximation provides quantitatively accurate analytic results for these chains.
\end{abstract}

DOI: 10.1103/PhysRevE.80.031303

PACS number(s): 45.70. $-\mathrm{n}, 46.40 . \mathrm{Cd}, 43.25 .+\mathrm{y}, 05.65 .+\mathrm{b}$

\section{INTRODUCTION}

The study of pulse propagation through granular media has attracted a great deal of attention for several reasons. Apart from addressing fundamental problems of pulse dynamics in the presence of highly nonlinear interactions, it also has direct practical application. Pulse propagation has mostly been studied in monodisperse one-dimensional chains of spherical granules. Nesterenko first showed $[1,2]$ that an initial impulse at an edge of a granular chain in the absence of precompression can result in solitary waves propagating through the medium. Since then, this system and variants thereof have been a testbed of extensive theoretical and experimental studies [3-21].

The chain model has recently been generalized to study the (sometimes profound) effects on pulse propagation of dissipation $[4,5,9,10]$ and of polydispersity in the structure and mass of the granules [8,14-19]. In particular, polydispersity is frequently introduced in a regular fashion such as in tapered chains (TCs), in which the size and/or mass of successive granules systematically decreases or increases. Polydispersity is also introduced by distributing masses randomly, by "decorating" chains with small masses regularly or randomly placed among larger masses, and by optimizing grain distribution for particular purposes. For example, considerable recent activity has focused on pulse propagation in "forward tapered chains" [15-19], with some results corroborated experimentally $[20,21]$. In these chains each granule is smaller than the preceding granule according to a systematic pattern. Among the main results of these studies is that the energy imparted to the first granule is redistributed among increasingly larger numbers of granules as the pulse propagates, thus attenuating the impulse on subsequent granules as time proceeds. This is a desirable behavior in the context of shock absorption. In any case, almost all known results are numerical and therefore predictively limited. While a few specific realizations have been experimentally verified, we have found few analytic results for pulse propagation in polydisperse systems [15]. Analytic work is in principle possible (albeit in practice often extremely difficult) if a continuum approximation is valid. This approach was first implemented successfully for monodisperse granular chains, where the resulting continuum equations can be (approximately) solved analytically $[1-3,5,7]$. Even when a continuum formulation is appropriate, in many cases the resulting equations cannot be solved $[9,10]$.

In this work we develop a converse approach which focuses on the fact that the propagating front in granular chains is often narrow. Our theory invokes a binary collision approximation, which leads to analytic expressions for various quantities that characterize the propagating pulse. In an earlier paper we introduced a binary collision approximation for the calculation of the pulse velocity in monodisperse chains [6]. Interestingly, although it is "opposite" to the continuum approximation, we found that the binary collision model produces results that also capture the dynamics of pulse propagation in such chains. Here we generalize this approach to forward and backward TCs, that is, to chains in which successive granules decrease in size or increase in size. The high quality of the approximation is ascertained by comparison with numerical integration of the full equations of motion for two tapering protocols. In one, the granular radius increases or decreases linearly (backward or forward "linearly" TC), while in the second the radius increases or decreases exponentially (backward or forward "exponentially" TC).

In Sec. II we introduce the granular chain model and lay out the binary approximation procedure and its caveats. The analytic results for the backward TCs and comparisons for these chains with numerical integration results are presented in Sec. III. The results and comparisons for the forward TCs are presented in Sec. IV. Section V provides a summarizing closure.

\section{THE MODEL AND THE BINARY APPROXIMATION}

We consider chains of granules all made of the same material of density $\rho$. When neighboring granules collide, they repel each other according to the power-law potential

$$
V=\frac{a}{n} r_{k}^{\prime}\left|y_{k}-y_{k+1}\right|^{n} \text {. }
$$

Here $y_{k}$ is the displacement of granule $k$ from its position at the beginning of the collision, and $a$ is a constant determined 
by Young's modulus and Poisson's ratio [22,23]. The exponent $n$ is $5 / 2$ for spheres (Hertz potential), which we use in this paper [23]. We have defined

$$
r_{k}^{\prime}=\left(\frac{2 R_{k}^{\prime} R_{k+1}^{\prime}}{R_{k}^{\prime}+R_{k+1}^{\prime}}\right)^{1 / 2},
$$

where $R_{k}^{\prime}$ is the principal radius of curvature of the surface of granule $k$ at the point of contact. When the granules do not overlap, the potential is zero. The equation of motion for the $k$ th granule is

$$
\begin{aligned}
M_{k} \frac{d^{2} y_{k}}{d \tau^{2}}= & a r_{k-1}^{\prime}\left(y_{k-1}-y_{k}\right)^{n-1} \theta\left(y_{k-1}-y_{k}\right) \\
& -a r_{k}^{\prime}\left(y_{k}-y_{k+1}\right)^{n-1} \theta\left(y_{k}-y_{k+1}\right),
\end{aligned}
$$

where $M_{k}=(4 / 3) \pi \rho\left(R_{k}^{\prime}\right)^{3}$. The Heaviside function $\theta(y)$ ensures that the elastic interaction between grains only exists if they are in contact. Initially the granules are placed along a line so that they just touch their neighbors in their equilibrium positions (no precompression), and all but the leftmost particle are at rest. The initial velocity of the leftmost particle $(k=1)$ is $V_{1}$ (the impulse). We define the dimensionless quantity

$$
\alpha \equiv\left[\frac{M_{1} V_{1}^{2}}{a\left(R_{1}^{\prime}\right)^{n+1 / 2}}\right]
$$

and the rescaled quantities $x_{k}, t, m_{k}$, and $R_{k}$ via the relations

$$
\begin{gathered}
y_{k}=R_{1}^{\prime} \alpha^{1 / n} x_{k}, \quad \tau=\frac{R_{1}^{\prime}}{V_{1}} \alpha^{1 / n} t, \\
R_{k}^{\prime}=R_{1}^{\prime} R_{k}, \quad M_{k}=M_{1} m_{k} .
\end{gathered}
$$

Equation (3) can then be rewritten as

$$
\begin{aligned}
m_{k} \ddot{x}_{k}= & r_{k-1}\left(x_{k-1}-x_{k}\right)^{n-1} \theta\left(x_{k-1}-x_{k}\right) \\
& -r_{k}\left(x_{k}-x_{k+1}\right)^{n-1} \theta\left(x_{k}-x_{k+1}\right),
\end{aligned}
$$

where a dot denotes a derivative with respect to $t$, and

$$
r_{k}=\left(\frac{2 R_{k} R_{k+1}}{R_{k}+R_{k+1}}\right)^{1 / 2} .
$$

The rescaled initial velocity is unity, i.e., $v_{1}(t=0)=1$. The velocity of the $k$ th granule in unscaled variables is simply $V_{1}$ times its velocity in the scaled variables.

The equations of motion can be integrated numerically, and we do so for our TCs. In each chain we observe that the initial impulse quickly settles into a propagating pulse whose front typically involves only three granules. In the backward TCs the narrow pulse is "clean" in the sense that granules behind it are reflected back and thus do not participate in forward energy propagation. In the forward TCs the narrow front carries a tail of moving granules behind it, but this tail does not affect the propagation dynamics of the narrow front. From the numerical integration results we are able to extract quantities such as the pulse peak location, amplitude, and velocity as functions of granule index and of time. These are the quantities to be compared with those obtained from the binary collision theory.
The binary collision approximation is based on the assumption that the transfer of energy along the chain occurs via a succession of two-particle collisions. First, particle $k$ $=1$ of unit velocity collides with initially stationary particle $k=2$, which then acquires a velocity $v_{2}$ and collides with stationary particle $k=3$, and so on. The velocities after each collision can easily be obtained from conservation of energy and momentum. The velocity $v_{k+1}$ of granule $k+1$ after the collision with granule $k$ is always positive, so this particle goes on to collide with the next one. On the other hand, the velocity of granule $k$ after the collision may be positive or negative depending on the direction of tapering of the chain (it would be zero for a monodisperse chain). From these velocities, a number of other results follow, which we outline here, but implement for particular chains in later sections.

After the collision of granules $k$ and $k+1$, the latter emerges with velocity

$$
v_{k+1}=\frac{2 v_{k}}{1+\frac{m_{k+1}}{m_{k}}} .
$$

This result can be implemented recursively to obtain

$$
v_{k}=\prod_{k^{\prime}=1}^{k-1} \frac{2}{1+\frac{m_{k^{\prime}+1}}{m_{k^{\prime}}}}
$$

To actually evaluate this product we need to implement a tapering protocol, cf. below.

We wish to use these results to calculate the time it takes the pulse to move along the chain and, more specifically, the time it takes the pulse to arrive at the $k$ th granule. For this purpose, it is convenient to introduce the difference variable

$$
z_{k}=x_{k}-x_{k+1} .
$$

The equation of motion for the difference variable is obtained by subtracting the equations of motion of the two granules during a collision, cf. Eq. (6),

$$
\begin{gathered}
\ddot{x}_{k}=-\frac{r_{k}}{m_{k}}\left(x_{k}-x_{k+1}\right)^{n-1}, \\
\ddot{x}_{k+1}=\frac{r_{k}}{m_{k+1}}\left(x_{k}-x_{k+1}\right)^{n-1},
\end{gathered}
$$

which directly leads to

$$
\mu_{k} \ddot{z}_{k}=-r_{k} z_{k}^{n-1}
$$

Here $\mu_{k}=m_{k} m_{k+1} /\left(m_{k}+m_{k+1}\right)$ is the reduced mass. Equation (12) is the equation of motion of a particle of mass $\mu_{k}$ in the potential $\left(r_{k} / n\right) z_{k}^{n}$ defined for $z_{k} \geq 0$. The initial condition $\dot{z}_{k}(0)$ is simply $v_{k}$ since the velocity of granule $k+1$ is zero before the collision. Therefore, from Eq. (9) we can write 


$$
\dot{z}_{k}(0)=\prod_{k^{\prime}=1}^{k-1} \frac{2}{\left(1+\frac{m_{k^{\prime}+1}}{m_{k^{\prime}}}\right)} .
$$

The energy conservation condition

$$
\frac{1}{2} \dot{z}_{k}^{2}(t)+\frac{r_{k}}{n \mu_{k}} z_{k}^{n}(t)=\frac{1}{2} \dot{z}_{k}^{2}(0)
$$

leads to

$$
\dot{z}_{k}(t)=\left(\dot{z}_{k}^{2}(0)-\frac{2 r_{k}}{n \mu_{k}} z_{k}^{n}(t)\right)^{1 / 2} .
$$

We say that the pulse arrives at granule $k$ when the velocity of granule $k$ surpasses that of granule $k-1$, and that it moves on to granule $k+1$ when the velocity of the $(k+1)$ st granule surpasses that of the $k$ th granule. The residence time $T_{k}$ on granule $k$ is the time that granule $k$ takes to transfer the pulse from $k-1$ to $k+1$, and is given by

$$
T_{k}=\int_{0}^{z_{k}^{\max }} \frac{d z_{k}}{\dot{z}_{k}}=\int_{0}^{z_{k}^{\max }} \frac{d z_{k}}{\left(\dot{z}_{k}^{2}(0)-\frac{2 r_{k}}{n \mu_{k}} z_{k}^{n}\right)^{1 / 2}},
$$

where $z_{k}^{\max }$ is the compression when the velocities of particles $k$ and $k+1$ are equal [which is also the maximum compression, cf. Eq. (14)],

$$
z_{k}^{\max }=\left(\frac{n \mu_{k}}{2 r_{k}} \dot{z}_{k}^{2}(0)\right)^{1 / n} .
$$

The integral can be performed exactly to yield

$$
T_{k}=\sqrt{\pi}\left(\frac{n \mu_{k}}{2 r_{k}}\right)^{1 / n}\left[\dot{z}_{k}(0)\right]^{(-1+2 / n)} \frac{\Gamma(1+1 / n)}{\Gamma(1 / n+1 / 2)} .
$$

Finally, the total time taken by the pulse to pass the $k$ th granule is obtained by summing $T_{k}$,

$$
t=\sum_{k^{\prime}=1}^{k} T_{k^{\prime}}
$$

For a monodisperse chain $\mu_{k}=1 / 2, r_{k}=1$ and $\dot{z}_{k}(0)=1$, and Eq. (18) reduces to the result obtained in [6]. To evaluate these times explicitly for tapered chains we need to specify a tapering protocol.

\section{BACKWARD TAPERED CHAINS}

We proceed to implement these results for various tapered chains of spherical granules $(n=5 / 2)$, starting with backward tapering in this section. Two forms of tapering are considered.

\section{A. Linearly tapered chains}

In backward linearly TCs the radii of the granules grow linearly as $R_{k}=1+S(k-1)$, where $S>0$ is a fixed parameter. The ratios of the radii and of the masses of two successive granules then are

$$
\begin{gathered}
\frac{R_{k}}{R_{k+1}}=1-\frac{S}{1+S k}, \\
\frac{m_{k}}{m_{k+1}}=\left(1-\frac{S}{1+S k}\right)^{3} .
\end{gathered}
$$

The ratio of the masses can be substituted into Eq. (9),

$$
v_{k}=\prod_{k^{\prime}=1}^{k-1} \frac{2}{1+\left(1-\frac{S}{1+S k^{\prime}}\right)^{-3}} .
$$

A large- $k$ limit can be evaluated by calculating the derivative of $\ln v_{k}$ with respect to $k$, retaining leading terms in a Taylor expansion when both $k$ and $S k \gg 1$, and exponentiating. This leads to the asymptotic scaling relation

$$
v_{k} \sim k^{-3 / 2} \text {. }
$$

The amplitude of the velocity pulse decreases with increasing grain number, as would be expected since the granules grow in size and mass.

The associated parameters for a backward linearly TC are given by

$$
\begin{gathered}
\mu_{k}=(1+S k)^{3}\left(\frac{\left(1-\frac{S}{1+S k}\right)^{3}}{1+\left(1-\frac{S}{1+S k}\right)^{3}}\right), \\
r_{k}=(1+S k)^{1 / 2}\left(\frac{1-\frac{S}{1+S k}}{1-\frac{S / 2}{1+S k}}\right)^{1 / 2} .
\end{gathered}
$$

Using Eqs. (22)-(24) in Eq. (18), we find that in the large- $k$ limit $T_{k}$ varies with $k$ according to

$$
T_{k} \sim k^{13 / 10} .
$$

The total time $t$ taken by the pulse to reach the $k$ th granule is obtained from Eq. (19). For large $k, t$ varies as

$$
t \sim k^{23 / 10} \text {. }
$$

This growth with $k$ again reflects the slowing down of the pulse as the granules become more massive. Using Eq. (26) in Eq. (22), the decay in time of the pulse amplitude is found to be

$$
v(t) \sim t^{-15 / 23}
$$

Finally, in order to compute the speed of the pulse in space, we note that at $t=0$ the position of each granule in the uncompressed chain is

$$
X_{0}(k)=S k^{2}+2 k(1-S)+S-2,
$$

and when the pulse has moved to the $k$ th granule its position is given by 


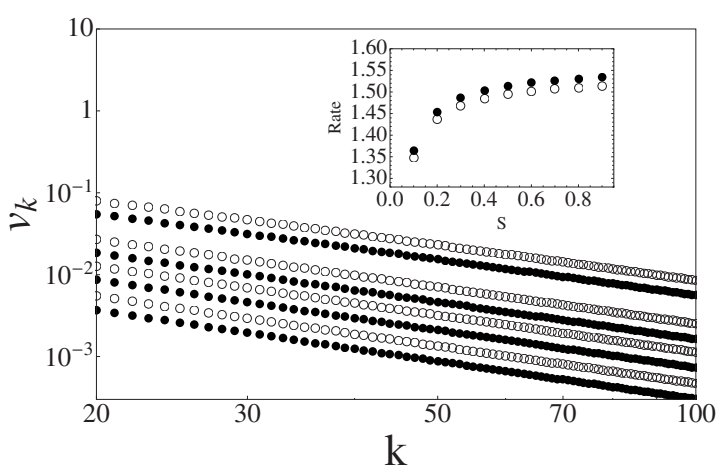

FIG. 1. The numerical integration data (solid circles) is compared with the binary collision approximation (open circles) for the decay of the velocity pulse amplitude with $k$ for backward linearly TCs with $S=0.2,0.4,0.6$, and 0.9 , from top to bottom. The inset shows the decay exponent, whose theoretical value approaches $3 / 2$. The numerical integration results also approach an $S$-independent value that is slightly larger than but close to the theoretical value.

$$
X(k, t)=X_{0}(k)+x_{k}(t) .
$$

For large $k$ values, the static contribution $X_{0}(k)$ dominates and the position of the pulse is

$$
X(t) \sim k^{2} \sim t^{20 / 23} .
$$

The speed $c(t)$ of the pulse therefore varies in time as

$$
c(t)=\frac{d X}{d t} \sim t^{-3 / 20} .
$$

Thus, the binary collision approximation in the backward linearly TC leads to a power-law dependence in both $k$, Eq. (22), and $t$, Eq. (27), for the pulse amplitude, for position (30), and for speed (31). Note that these asymptotic decays are independent of the tapering parameter $S$.

The comparison of the predictions of the binary collision approximation with the numerical integration of Eq. (6) is carried out as follows. First, consider the decay of the amplitude of the velocity pulse as a function of $k$. In Fig. 1 we

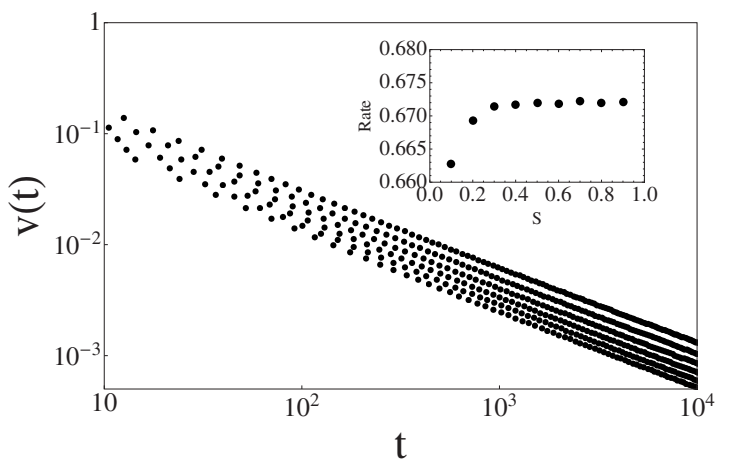

FIG. 2. The decay of the velocity pulse amplitude with time for backward linearly TCs with $S=0.4$ to 0.9 in steps of 0.1 from top to bottom, as obtained from numerical integration. The inset shows the rate of decay for different $S$ values. The long-time binary prediction is $15 / 23=0.652 \ldots$, within $2 \%-3 \%$ of the numerical integration results.

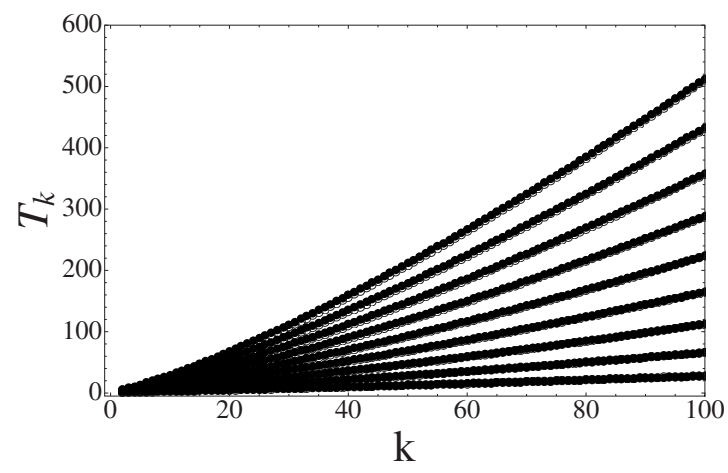

FIG. 3. Residence time of the pulse on granule $k$ for backward linearly TCs. Comparison between the binary collision approximation (open circles, barely visible because of the agreement between theory and numerical integration) and direct numerical integration of the equations of motion (solid circles). From bottom to top, $S$ $=0.1$ to 0.9 in steps of 0.1 .

show $v_{k}$ vs $k$ from numerical integration of the equations of motion (solid circles) and as calculated from the binary collision theory, Eq. (21) (open circles). The comparison is made for various values of $S$, as indicated in the caption. Note that in some regimes of this figure the decay of the pulse amplitude is $S$-dependent, as found in Eq. (21) and also from the numerical integration; the $S$-independent power-law decay (22) is only valid when $S k \gg 1$. Figure 1 reveals the single weakest aspect of the theory in that it shows substantial differences in the absolute magnitude of the pulse amplitude between the theoretical and numerical integration results, but the rate of decay of $v_{k}$ is captured very well, within $\approx 1 \%-2 \%$, as emphasized by the inset. The inset also shows the approach to the $S$-independent power $3 / 2$ for large $k$, as predicted in Eq. (22).

The decay of the velocity pulse in time as obtained from numerical integration is shown in Fig. 2. The power-law decay (27) predicted from the binary theory is in excellent agreement with the numerical data. In the inset we show the power-law exponent for different $S$ values. The long-time value of the exponent $15 / 23=0.652 \ldots$ obtained from the

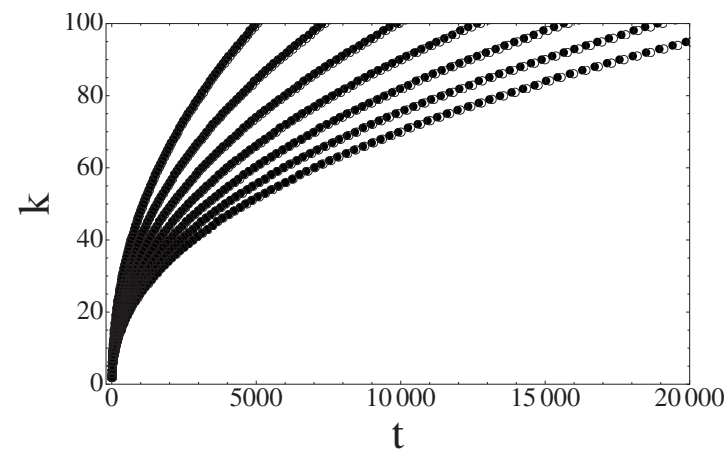

FIG. 4. Position of the pulse in units of grains versus time for backward linearly TCs. Comparison between the binary collision approximation (open circles, barely visible because of the agreement between theory and numerical integration) and direct numerical integration of the equations of motion (solid circles). From top to bottom, $S=0.3$ to 0.9 in steps of 0.1 . 
TABLE I. Backward linearly TCs: Values of $b$ obtained from the binary collision approximation and from the numerical integration of the equations of motion in the asymptotic expression $k \sim t^{b}$ for the position of the pulse (in units of grains) as a function of time. The theoretical asymptotic value of $b$ is $10 / 23=0.435 \ldots$

\begin{tabular}{ccc}
\hline \hline$S$ & Theory & Numerics \\
\hline 0.3 & 0.439 & 0.438 \\
0.4 & 0.438 & 0.437 \\
0.5 & 0.437 & 0.436 \\
0.6 & 0.436 & 0.436 \\
0.7 & 0.436 & 0.436 \\
0.8 & 0.436 & 0.435 \\
0.9 & 0.436 & 0.435 \\
\hline
\end{tabular}

theory is in very good agreement (within 2\%-3\%) with the observed data. Furthermore, gratifying agreement is found if we compare the time $T_{k}$ obtained from Eq. (18) with numerical integration results, as seen in Fig. 3. The time $t$ that the pulse takes to pass by granule $k$ is also very well reproduced by the theory, cf. Fig. 4.

In Table I the agreement between the theory and the integration results is made explicit. We list the results of fitting numerical integration data for much longer chains $(N$ $>400$ ) with a power-law form $k=a t^{b}$. The numerical uncertainties (standard deviations) are in the third decimal place. For small values of $S$ the data does not reach the values of $k$ that are needed for the asymptotic behavior $t^{10 / 23}$ of Eq. (26) to be valid $(S k \gg 1)$. For the larger values of $S$ the asymptotic behavior is approached, and here the agreement with the predicted asymptotic value $b=10 / 23=0.435 \ldots$ becomes evident. Since Eq. (26) is valid only in the large- $k$ regime, in these fits we have only used the information for granules $k$ $=300$ to $k=400$.

\section{B. Exponentially tapered chains}

A backward exponentially TC is one in which the ratio of radii of successive granules is constant,

$$
\frac{R_{k}}{R_{k+1}}=1-q,
$$

where $0<q<1$. The radii of granules thus increase in geometric progression. The radius of the $k$ th granule is $R_{k}=(1$ $-q)^{1-k}$, and the mass ratio of two consecutive granules is

$$
\frac{m_{k}}{m_{k+1}}=(1-q)^{3}
$$

This is the case that has been considered in many previous studies [15-19]. This mass ratio in Eq. (9) leads to the velocity amplitude

$$
v_{k}=A(q) e^{-k \ln A(q)},
$$

where

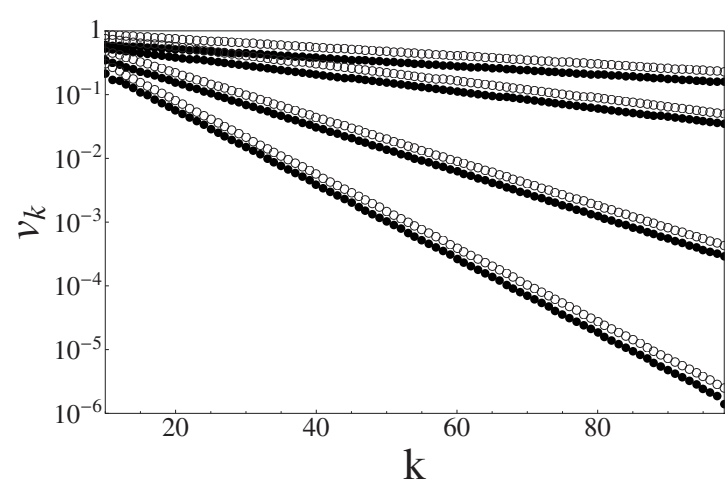

FIG. 5. The numerical data (solid circles) is compared with the binary collision approximation (open circles) for the decay of the velocity pulse amplitude in backward exponentially TCs for $q$ $=0.01,0.02,0.05$, and 0.08 from top to bottom.

$$
A(q)=\frac{1}{2}\left[1+(1-q)^{-3}\right]
$$

and to the associated parameters

$$
\begin{gathered}
\mu_{k}=\frac{(1-q)^{-3(k-1)}}{\left[1+(1-q)^{3}\right]}, \\
r_{k}=(1-q / 2)^{-1 / 2}(1-q)^{-(k-1) / 2} .
\end{gathered}
$$

Substituting Eqs. (34), (36), and (37) in Eq. (18) and following the steps implemented for the linearly TC we find that the time $t$ for the pulse to reach the $k$ th granule now grows exponentially with $k$ for large $k$,

$$
t \sim e^{\eta(q) k}
$$

where

$$
\eta(q)=\ln \left(\frac{[A(q)]^{1 / 5}}{1-q}\right) .
$$

Combining Eq. (34) with Eq. (38) leads to the decay of the pulse amplitude as a function of time,

$$
v(t) \sim t^{-f(q)},
$$

with

$$
f(q)=\frac{1}{\eta(q)} \ln A(q) .
$$

The position of the $k$ th granule at time $t$ is

$$
X(k, t)=\frac{2-q}{q}\left[(1-q)^{-k+1}-1\right]+x_{k}(t) .
$$

For large $k$, we can again ignore the contribution due to the displacement compared to the dominant static part. This allows us to approximate the pulse position as

$$
X(k) \sim \frac{2-q}{q(1-q)} e^{-k \ln (1-q)},
$$

which implies that 
TABLE II. Exponential rates of decay for the velocity pulse in $k$ space for backward exponentially TCs as obtained from the binary collision approximation $[\ln A(q)$ in Eq. (34)] and the numerical integration of Eq. (6).

\begin{tabular}{ccc}
\hline \hline$q$ & Theory & Numerics \\
\hline 0.01 & 0.01519 & 0.01522 \\
0.02 & 0.03076 & 0.03088 \\
0.03 & 0.04673 & 0.04697 \\
0.04 & 0.06311 & 0.06350 \\
0.05 & 0.07990 & 0.08047 \\
0.06 & 0.09711 & 0.09788 \\
0.07 & 0.11477 & 0.11568 \\
0.08 & 0.13287 & 0.13378 \\
0.09 & 0.15144 & 0.15275 \\
\hline \hline
\end{tabular}

$$
X(t) \sim t^{1-f(q) / 5}
$$

and hence the pulse velocity in time decays as

$$
c(t) \sim t^{-f(q) / 5} .
$$

Thus, for backward exponentially TCs the pulse amplitude and position vary exponentially in $k$, but in time the variations are power laws.

Figure 5 shows the exponential decay of the pulse amplitude with $k$ as predicted from the theory, Eq. (34), and as obtained from direct numerical integration of the equations of motion. We find a small difference between the amplitudes predicted by theory and those obtained from the numerical integration, but the predicted decay rate is again in excellent agreement with the actual data. We compare the decay rates for each $q$ value obtained by fitting the numerical data in Fig. 5 with those calculated from the theory. The results are shown in Table II.

The power-law decay of the pulse amplitude in time is shown in Fig. 6. The variations in the data points for the largest value of the tapering parameter have no physical significance and are of purely numerical origin. The rates of decay are close to those predicted by the theory, Eq. (40). In Table III we compare the two results for different $q$ values. Note that the asymptotic expressions are valid only in the long-time limit, a requirement that involves longer times as

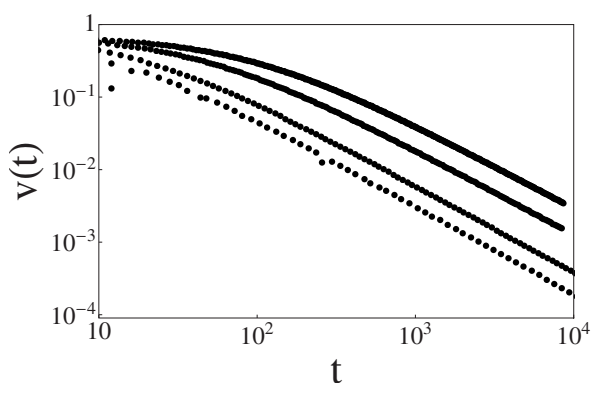

FIG. 6. Decay of the pulse amplitude with time for backward exponentially TCs obtained from numerical integration for $q$ $=0.01,0.02,0.05$, and 0.08 from top to bottom.
TABLE III. Asymptotic rates of decay for the pulse amplitude in time for backward exponentially TCs obtained from the binary collision approximation $[f(q)$ in Eq. (41)] and those obtained by fitting the straight-line portions of the data in Fig. 6.

\begin{tabular}{ccc}
\hline \hline$q$ & Theory & Numerics \\
\hline 0.01 & 1.1605 & 1.1279 \\
0.02 & 1.1673 & 1.1546 \\
0.03 & 1.1740 & 1.1744 \\
0.04 & 1.1808 & 1.1820 \\
0.05 & 1.1877 & 1.1803 \\
0.06 & 1.1945 & 1.1952 \\
0.07 & 1.2015 & 1.2049 \\
0.08 & 1.2084 & 1.2145 \\
0.09 & 1.2154 & 1.2256 \\
\hline
\end{tabular}

the value of $q$ decreases. The small differences in the results arise mainly because Eq. (40) is a long-time limit (and not because of the binary approximation per se).

Finally, the theoretical residence times $T_{k}$ and the time $t$ for the pulse to pass through the $k$ th granule are in excellent agreement with the numerical integration data, as can be seen in Figs. 7 and 8.

\section{FORWARD TAPERED CHAINS}

Next we consider chains where the granules are successively smaller starting from the granule that is the subject of the initial impulse, that is, chains with forward tapering. We present our binary collision approximation results and compare them with the results obtained from the numerical integration of the full equations of motion [Eq. (6)]. We again consider two different tapering protocols, the linear and the exponential.

\section{A. Linearly tapered chains}

In forward linearly TCs the radii of the granules decrease linearly as $R_{k}=1-S(k-1)$. Note that since the radius of the

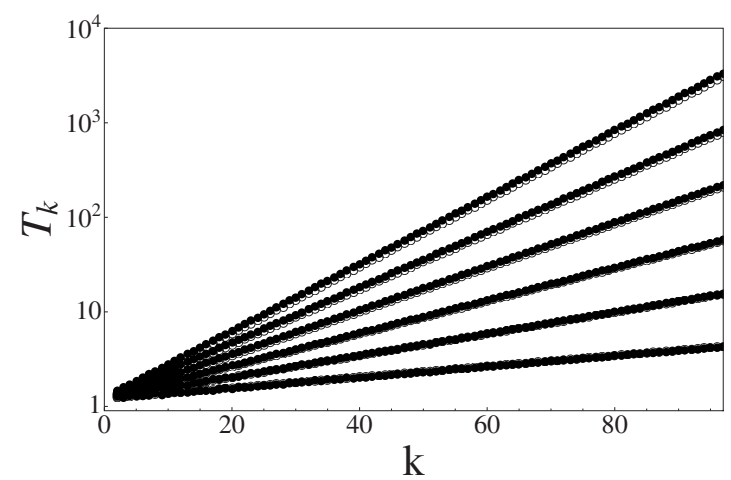

FIG. 7. The pulse residence time $T_{k}$ as a function of $k$ for backward exponentially TCs is shown for $q=0.01$ to 0.06 in steps of 0.01 (top to bottom). Theory and numerical results are denoted by open circles and filled circles, respectively. 


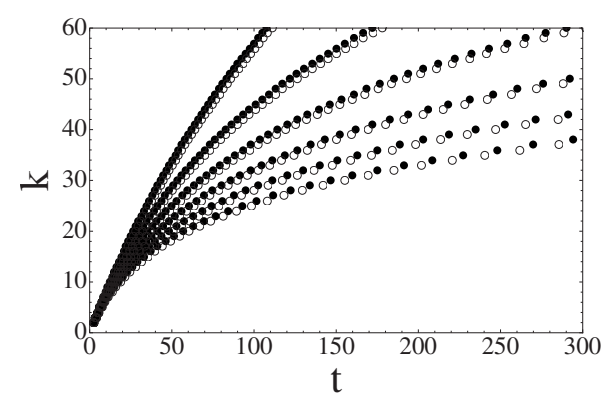

FIG. 8. The position of the pulse in units of grains versus time $t$ for backward exponentially TCs is shown for $q=0.01$ to 0.06 in steps of 0.01 (top to bottom). Theory and numerical results are denoted by open circles and filled circles, respectively.

first granule is unity, this places an $S$-dependent restriction on the chain length $N$ (or an $N$-dependent restriction on the tapering parameter $S$ ),

$$
N<1+\frac{1}{S}
$$

In our calculations we set $N=100$, and accordingly restrict the value of $S$ to be $\leq 0.01$. The ratios of the radii and of the masses of the $k$ th to $(k+1)$ st granules are

$$
\begin{gathered}
\frac{R_{k}}{R_{k+1}}=1+\frac{S}{1-S k}, \\
\frac{m_{k}}{m_{k+1}}=\left(1+\frac{S}{1-S k}\right)^{3} .
\end{gathered}
$$

Substituting Eq. (47) in Eq. (9), we obtain

$$
v_{k}=\prod_{k^{\prime}=1}^{k-1} \frac{2}{1+\left(1+\frac{S}{1-S k^{\prime}}\right)^{-3}} .
$$

When $k \ll 1+1 / S$ we follow the steps described in going from Eq. (21) to Eq. (22) to find the scaling behavior

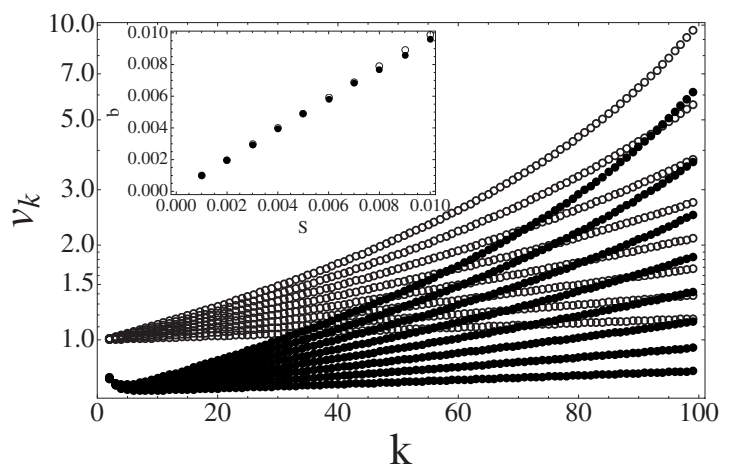

FIG. 9. The numerical integration data (filled circles) is compared with the binary collision approximation results (open circles) for the velocity pulse amplitude in forward linearly TCs for $S$ from 0.001 to 0.008 in steps of 0.001 (bottom to top). The inset shows the variation in the parameter $b$ with $S$ in the fit of $v_{k}$ described in the text.

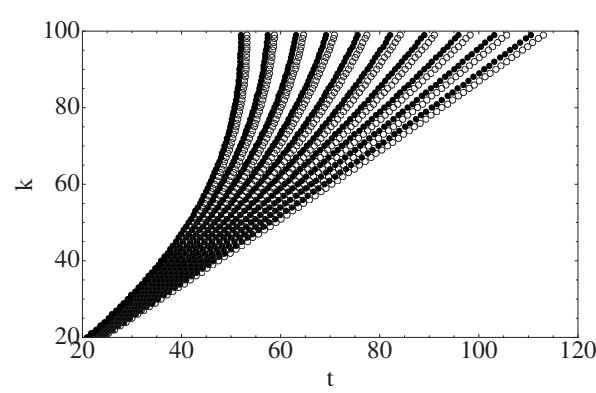

FIG. 10. Comparison of the theory (open circles) and numerical integration results (filled circles) for the time $t$ taken by the pulse in forward linearly TCs to reach the $k$ th granule for $S$ from 0.001 to 0.01 in steps of 0.001 from right to left.

$$
v_{k} \sim\left(1-\frac{k}{1+1 / S}\right)^{-3 / 2} .
$$

Since $k<1+1 / S$, this function grows as $k$ increases. This is expected since grain mass decreases with increasing $k$. The associated parameters $\mu_{k}$ and $r_{k}$ for forward linear TCs are obtained by replacing $S$ with $-S$ in Eqs. (23) and (24). Using Eq. (50) in Eq. (18) and summing over $k$, we obtain the scaling for the time it takes the pulse to pass through the $k$ th granule,

$$
t \sim C(S)\left[1-(1+S)^{23 / 10}\left(1-\frac{k}{1+1 / S}\right)^{23 / 10}\right],
$$

where $C(S)=C_{0}(S)[1+\mathcal{O}(S)]$ and

$$
C_{0}(S)=\frac{10 \sqrt{\pi}}{23}\left(\frac{5}{8}\right)^{2 / 5} \frac{\Gamma(7 / 5)}{\Gamma(9 / 10)}\left(1+\frac{1}{S}\right) .
$$

Figure 9 shows the variation in the pulse amplitude with $k$ as obtained from the theory [Eq. (49)] and the numerical integration.

As predicted from Eq. (50), we observe a linear growth in the pulse amplitude for small $k$. Also, as $k \rightarrow N$ the numerical results show that the velocity behaves as $v_{k} \sim a(1-b k)^{-3 / 2}$ and thus diverges around $k \sim 1 / b$. Interestingly, we find that $1 / b$ is very close to $(1+1 / S)$ and thus Eq. (50), which is in

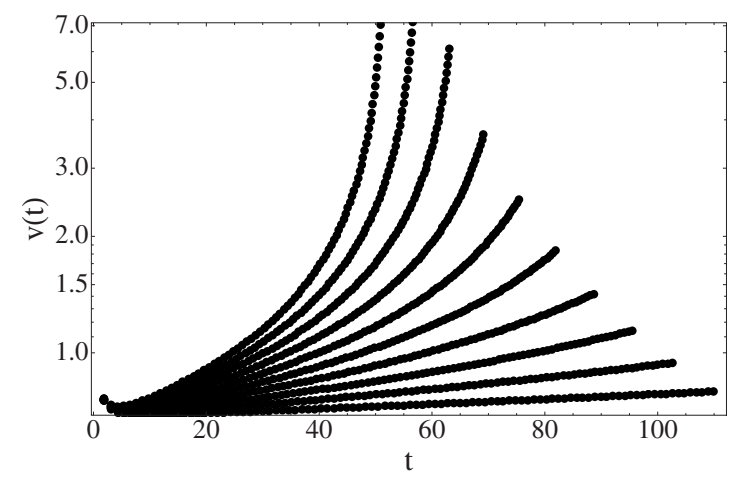

FIG. 11. Change in the pulse amplitude with time for forward linearly TCs as obtained from numerical integration of the equations of motion. Results are for $S$ from 0.001 to 0.01 in steps of 0.001 from bottom to top. 


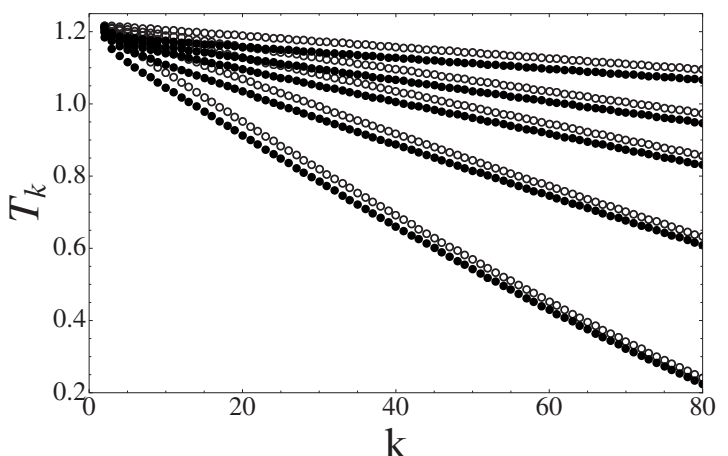

FIG. 12. Residence time of the pulse on each granule for forward linearly TCs for $S=0.001,0.002,0.003,0.005$, and 0.009 from top to bottom. Numerical integration results: filled circles; binary collision approximation results: open circles.

principle restricted to the regime $k \ll 1+1 / S$, seems to work well all the way up to the divergence. In the inset in Fig. 9 we compare the values of $b$ obtained from the theory, $b$ $=(1+1 / S)^{-1}$, and the fit to numerical data.

In Fig. 10 we compare the results for the time $t$ taken by the pulse to pass through the $k$ th granule. The change in $t$ with $k$ is described extremely accurately by the binary collision approximation result [Eq. (51)] valid for $k \ll 1 / S$. The result in Eq. (51) is thus expected to be valid for all $k$ provided that $S$ is small $(1 / S \gg 1)$. Interestingly, the result in Eq. (51) in fact works well for all $k \leq N$ (with $N=100$ ) in the range $0.001 \leq S \leq 0.01$. The time $t$ increases linearly with $k$ for small $k$ but becomes almost $k$-independent as $k$ increases. This is a reflection of the fact that the granular mass and size become very small and as a result the pulse passes through these grains very quickly.

The change in the pulse amplitude with time is shown in Fig. 11. The theoretical result for the time-dependent amplitude can be obtained by combining Eq. (50) with Eq. (51). We find that

$$
v(t) \sim\left(1-\frac{t}{C(S)}\right)^{-15 / 23} .
$$

For small $t$ the pulse amplitude thus grows linearly with $t$, and as $t \rightarrow C(s)$ it diverges with exponent $15 / 23$. Since the

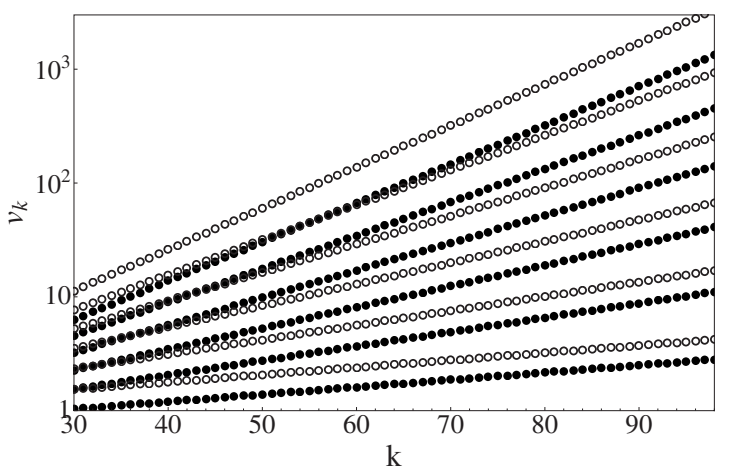

FIG. 13. Change in the pulse amplitude as a function of $k$ for backward exponentially TCs for $q$ from 0.01 to 0.06 in steps of 0.01 from bottom to top. Numerical integration results: filled circles. Binary collision approximation results: open circles.
TABLE IV. Comparison of the rates for the exponential growth of the pulse amplitude in forward exponentially TCs in granule space obtained from the binary collision approximation with those obtained by fitting the data in Fig. 13.

\begin{tabular}{ccc}
\hline \hline$q$ & Theory & Numerics \\
\hline 0.01 & 0.01481 & 0.01471 \\
0.02 & 0.02926 & 0.02879 \\
0.03 & 0.04336 & 0.04267 \\
0.04 & 0.05710 & 0.05565 \\
0.05 & 0.06848 & 0.07051 \\
0.06 & 0.08359 & 0.07833 \\
\hline \hline
\end{tabular}

absolute value of the pulse amplitude is not captured correctly by the binary collision approximation, the divergence time $C(S)$ of the pulse amplitude is also different from the actual divergence time seen in the numerics. However, we find that the initially linear behavior and the divergence exponent 15/23 are accurately predicted by the theory. In Fig. 12 we show the pulse residence time obtained for each granule from numerical integration along with the corresponding values obtained from the binary collision approximation.

\section{B. Exponentially tapered chains}

In this case the radii of the granules decrease as

$$
R_{k}=\frac{1}{(1+q)^{k-1}},
$$

where $q>0$. The velocity of the $k$ th granule can be obtained from Eq. (34) by replacing $q$ by $-q$. Figure 13 shows the comparison of the binary collision approximation results with the numerical data. We again observe that although the absolute values differ by a constant amount, the rate of growth is correctly predicted by the theory. In Table IV we compare the rates obtained from the fit to the data points in Fig. 13 with the results of the theory.

The total time taken by the pulse to reach the $k$ th granule is obtained by substituting Eqs. (34) and (36) in Eq. (18), replacing $q$ by $-q$, and summing over $k$, cf. Eq. (19). For long times, we obtain

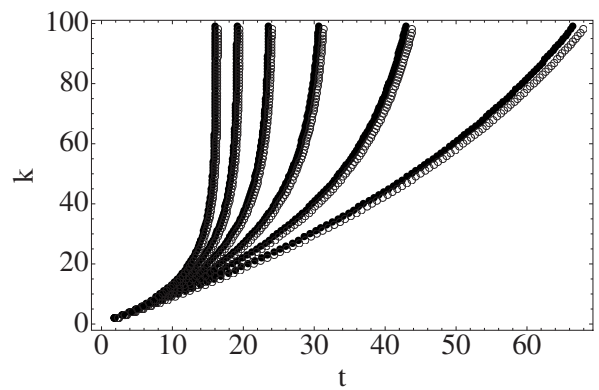

FIG. 14. Total time taken by the pulse to pass through the $k$ th granule in forward exponentially TCs for $q$ from 0.01 to 0.06 in steps of 0.01 (right to left). Numerical integration results: filled circles. Binary collision approximation results: open circles. 


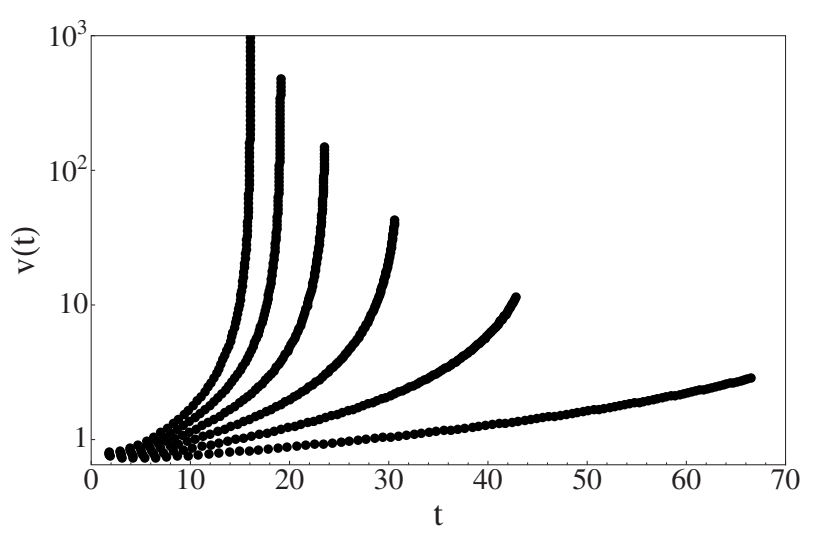

FIG. 15. Numerical integration results for the pulse amplitude as a function of time in forward exponentially TCs for $q$ from 0.01 to 0.06 in steps of 0.01 (bottom to top).

$$
t \sim 1-e^{(k-1) \eta(-q)}
$$

where $\eta(-q)$ is obtained by replacing $q$ by $-q$ in Eq. (39). Since $\eta(-q)<0, t$ approaches a constant value at large $k$. In Fig. 14 we show both the theory and the numerical results for the time taken by the pulse to pass through the $k$ th granule.

The time dependence of the pulse amplitude in the binary collision approximation is obtained as

$$
v(t) \sim\left(1+\frac{t}{M(q)}\right)^{-f(-q)},
$$

where

$$
M(q)=\frac{2 \sqrt{\pi}}{5 \eta(-q)} \frac{\Gamma(7 / 5)}{\Gamma(9 / 10)}\left(\frac{5 \sqrt{1-q / 2}}{4\left[1+(1+q)^{3}\right]}\right)^{2 / 5},
$$

and $f(-q)$ is obtained by replacing $q$ with $-q$ in Eq. (41). At short times $v(t)$ increases linearly with $t$. Also, since $\eta(-q)$ $<0, M(q)<0$ and, as $t \rightarrow M(q)$, the velocity diverges with the exponent $f(-q)$. These behaviors are shown in Fig. 15 . Again, since the absolute value of the pulse amplitude is not captured correctly within the binary collision approximation, the divergence time of the pulse amplitude $M(q)$ is also different from the actual divergence time seen in the numerics. However, the exponent $f(-q)$ is accurately predicted by the theory. In Table $\mathrm{V}$ we compare the exponent $f(-q)$ obtained by fitting the numerical data in Fig. 15 with the results of the theory.

\section{CONCLUSIONS}

We have introduced an analytic approach to calculate the behavior of a propagating pulse along a variety of tapered chains of spherical granules. We implement a binary collision approximation [6] that supposes that the collision events by which a pulse propagates involve only two granules at a time. While the approximation overestimates the pulse amplitude, it captures all other properties remarkably well.
TABLE V. Comparison of the power-law exponent $f(-q)$, Eq. (56), obtained from the binary collision approximation with the exponent extracted from numerical integration results for forward exponentially TCs.

\begin{tabular}{ccc}
\hline \hline$q$ & Theory & Numerics \\
\hline 0.01 & 1.1472 & 1.1361 \\
0.02 & 1.1406 & 1.1308 \\
0.03 & 1.1341 & 1.1134 \\
0.04 & 1.1276 & 1.1237 \\
0.05 & 1.1211 & 1.1022 \\
0.06 & 1.1147 & 1.1101 \\
\hline \hline
\end{tabular}

These include the rate of decay of the pulse amplitude with time, the residence time of the pulse on each granule, and the pulse speed in units of granule and in space. We take note of other binary collision schemes in the literature. In one [15], the granular collisions are described by a hard sphere potential. In another [24], the object was to study the effects of viscoelastic interactions by assigning a velociry-dependent coefficient of restitution to the granular collisions.

We have implemented our approximation in four different tapered chains: backward linearly and exponentially tapered chains (the granules increase in size), and forward linearly and exponentially tapered chains (the granules decrease in size). In backward linearly tapered chains, where granular size increases linearly along the direction of pulse propagation, an initial impulse on the first granule settles into a pulse whose amplitude, speed, and width change slowly as the pulse propagates. The pulse speed and the pulse amplitude decay in granular units and also in real time, as is reasonable since the granules increase in size and mass along the direction of propagation. Although the pulse remains narrow in granule number, the pulse width in real space of course increases as the granules get larger. All these decreases and increases vary as power laws both in granular units $k$ and in time $t$ for large $k$ and $t$, and the decay and growth exponents are insensitive to the value of the tapering $S$ over the range that we have tested, $0.1<S \leq 1$. On the other hand, for backward exponentially tapered chains, where granule size increases geometrically, the pulse properties change exponentially in $k$ while they exhibit power-law behavior in time. In this case the rates are strongly influenced by the tapering parameter $q$.

In forward linearly tapered chains, where granular size decreases linearly, the pulse speeds up both in granule number units and in time, and in fact diverges. The divergence exponents are again insensitive to the tapering parameter $S$ and are quantitatively reproduced by the theory. Similar divergences are observed in the forward geometrically tapered chain, but now the behavior is sensitive to the tapering parameter $q$.

In the binary collision approximation the initial velocity for the collision between granules $k$ and $k+1$ is taken to be the velocity of granule $k$ at the end of the collision between 
granules $k-1$ and $k$. This estimate in turn leads to a pulse velocity amplitude that is higher than that obtained from the numerical integration of the full equations of motion, but all other pulse characteristics are quantitatively reproduced in all our tapered chains by this analytic approximation. Our next challenge is to generalize this approach to chains with other mass variation profiles and even to random chains. This work is in progress.

\section{ACKNOWLEDGMENTS}

Acknowledgment is made to the Donors of the American Chemical Society Petroleum Research Fund for partial support of this research (K.L. and U.H.). A.R. acknowledges support from Pronex-CNPq-FAPESQ and CNPq. M. E. is supported by the FNRS Belgium (chargé de recherches) and by the government of Luxembourg (Bourse de formation recherches).
[1] V. F. Nesterenko, J. Appl. Mech. Tech. Phys. 24, 733 (1983).

[2] A. N. Lazaridi and V. F. Nesterenko, J. Appl. Mech. Tech. Phys. 26, 405 (1985).

[3] V. F. Nesterenko, Dynamics of Heterogeneous Materials (Springer, New York, 2001).

[4] S. Job, F. Melo, A. Sokolow, and S. Sen, Phys. Rev. Lett. 94, 178002 (2005).

[5] A. Rosas and K. Lindenberg, Phys. Rev. E 68, 041304 (2003).

[6] A. Rosas and K. Lindenberg, Phys. Rev. E 69, 037601 (2004).

[7] E. J. Hinch and S. Saint-Jean, Proc. R. Soc. London, Ser. A 455, 3201 (1999).

[8] P. J. Wang, J. H. Xia, Y. D. Li, and C. S. Liu, Phys. Rev. E 76, 041305 (2007).

[9] A. Rosas, A. H. Romero, V. F. Nesterenko, and K. Lindenberg, Phys. Rev. Lett. 98, 164301 (2007).

[10] A. Rosas, A. H. Romero, V. F. Nesterenko, and K. Lindenberg, Phys. Rev. E 78, 051303 (2008).

[11] C. Coste, E. Falcon, and S. Fauve, Phys. Rev. E 56, 6104 (1997).
[12] V. F. Nesterenko, J. Phys. IV 4, C8-729 (1994).

[13] A. Sokolow, E. G. Bittle, and S. Sen, EPL 77, 24002 (2007)

[14] S. Sen, Phys. Rep. 462, 21 (2008).

[15] R. Doney and S. Sen, Phys. Rev. Lett. 97, 155502 (2006).

[16] D. T. Wu, Physica A 315, 194 (2002).

[17] R. L. Doney and S. Sen, Phys. Rev. E 72, 041304 (2005).

[18] A. Sokolow, J. M. M. Pfannes, R. L. Doney, M. Nakagawa, J. H. Agui, and S. Sen, Appl. Phys. Lett. 87, 254104 (2005).

[19] S. Sen, F. S. Manciu, and M. Manciu, Physica A 299, 551 (2001).

[20] F. Melo, S. Job, F. Santibanez, and F. Tapia, Phys. Rev. E 73, 041305 (2006).

[21] S. Job, F. Melo, A. Sokolow, and S. Sen, Granular Matter 10, 13 (2007).

[22] L. D. Landau and E. M. Lifshitz, Theory of Elasticity (Addison-Wesley, Massachusetts, 1959).

[23] H. Hertz, J. Reine Angew. Math. 92, 156 (1881).

[24] T. Pöschel and N. V. Brilliantov, Phys. Rev. E 63, 021505 (2001). 